\title{
Image-based Monitoring for Early Detection of Fouling in Crystallisation Processes
}

\author{
Christos Tachtatzis ${ }^{\mathrm{a}}$, Rachel Sheridan ${ }^{\mathrm{b}}$, Craig Michie ${ }^{\mathrm{a}}$, \\ Robert C. Atkinson ${ }^{\mathrm{a}}$, Alison Cleary ${ }^{\mathrm{a}}$, Jerzy Dziewierz ${ }^{\mathrm{c}}$, Ivan Andonovic ${ }^{\mathrm{a}}$, \\ Naomi Briggs $^{\mathrm{d}}$, Alastair J. Florence ${ }^{\mathrm{d}}$, Jan Sefcik ${ }^{\mathrm{b}, *}$ \\ ${ }^{a}$ Centre for Intelligent Dynamic Communications, \\ Department of Electronic and Electrical Engineering, \\ University of Strathclyde, Glasgow, Scotland, G1 $1 X W$, UK \\ ${ }^{b}$ EPSRC Centre for Innovative Manufacturing in \\ Continuous Manufacturing and Crystallisation, \\ Department of Chemical and Process Engineering, \\ University of Strathclyde, Glasgow, Scotland, G1 1XJ, UK \\ ${ }^{c}$ Centre for Ultrasonic Engineering, \\ Department of Electronic and Electrical Engineering, \\ University of Strathclyde, Glasgow, Scotland, G1 1 XW, UK \\ ${ }^{d}$ EPSRC Centre for Innovative Manufacturing in \\ Continuous Manufacturing and Crystallisation, \\ Strathclyde Institute of Pharmacy and Biomedical Sciences, \\ University of Strathclyde, Glasgow, Scotland, G4 ORE, UK
}

\section{Abstract}

Fouling or encrustation is a significant problem in continuous crystallisation processes where crystal deposits at surfaces impede heat transfer, increase flow resistance and reduce product quality. This paper proposes an automatic algorithm to detect early stages of fouling using images of vessel surfaces

\footnotetext{
*Corresponding author

Email addresses: christos.tachtatzis@strath.ac.uk (Christos Tachtatzis), rachel.sheridan@strath.ac.uk (Rachel Sheridan), c.michie@strath.ac.uk (Craig Michie), robert.atkinson@strath.ac.uk (Robert C. Atkinson), alison.cleary@strath.ac.uk (Alison Cleary), jerzy.dziewierz@strath.ac.uk (Jerzy Dziewierz), i.andonovic@strath.ac.uk (Ivan Andonovic), naomi.briggs@strath.ac.uk (Naomi Briggs), alastair.florence@strath.ac.uk (Alastair J. Florence), jan.sefcik@strath.ac.uk (Jan Sefcik)
} 
from commodity cameras. Statistical analysis of the pixel intensity variation offers the ability to distinguish appearance of crystals in the bulk solution and on the crystalliser walls. This information is used to develop a fouling metric indicator and determine separately induction times for appearance of first crystals at the surfaces and in the bulk. A method to detect process state changes using Bayesian online change point detection is also proposed, where the first change point is used to determine induction time either at the surface or in the bulk, based on real-time online measurements without using any predetermined threshold which usually varies between experiments and depends on data acquisition equipment. This approach can be used for in situ monitoring of early signs of encrustation to allow early warning for corrective actions to be taken when operating continuous crystallisation processes.

Keywords:

Continuous Crystallisation, Fouling, Encrustation, Nucleation, Induction Time, Imaging, Change Point Detection

\section{Introduction}

2 Crystallisation is an important unit operation in chemical, pharmaceu3 tical and food industries for isolation and purification of intermediates and 4 final products. Fouling or encrustation in the context of crystallisation is the 5 formation of crystal deposits on equipment surfaces, pipe walls and process 6 analytical probes and occurs as a result of heterogeneous nucleation and/or 7 attachment of crystals and their subsequent growth at solid surfaces $[1,2]$. 8 Fouled surfaces impede heat transfer [3], increase flow resistance and de9 crease product quality [4]. The impact of fouling and encrustation is even 
greater on continuous crystallisation processes where system blockages result in shutdowns leading to losses of time and revenue. Process conditions that influence fouling in crystallisation processes include solvent composition, supersaturation, $\mathrm{pH}$, temperature, flow velocity and regime, additives or impurities, while interface conditions that affect fouling include surface energy, roughness and topography, number of nucleation sites and aging of the fouling layer [5]. Mechanisms of initiating encrustation have been studied previously, where a crystalline phase was nucleated on solid surfaces at controlled local supersaturation and without influence from particle attachment [6].

Common methods to mitigate fouling include mechanically altering the surface using methods such as ultrasound or scraping or chemically altering the surface using coatings to reduce the number of nucleation sites and/or remove attached crystals [7]. Increasing the induction time for surface fouling can be achieved by changing the energy and topography of the surface or by changing the flow conditions [8]. The rough surface can stabilising crystal nuclei and promote growth on the surface, while fouling is typically delayed at smoother surfaces.

A review of detection methods of fouling in the food industry can be found in previous literature [4]. Common, non-invasive methods to detect fouling are to monitor the hydrodynamic and heat transfer parameters. Hydrodynamic methods monitor the inlet and outlet pressures and infer the degree of deposits on the walls of a tube. Heat transfer methods monitor heat transfer losses to infer the degree of fouling. Both of these methods have low sensitivity and generally not suitable for early stage monitoring of 
fouling in continuous crystallisers since significant fouling must occur before temperature or pressure variations are detectable. Other methods include the measurement of the electrical resistance or conductivity, ultrasound and vibration, however all these alter the fouling (i.e. fouled deposits may be broken off and then drift downstream). In the crystallisation domain it is common to use reflectance, turbidity and other spectroscopic methods to monitor processes in situ but none of these are specifically suited to provide direct information about the state of vessel walls and presence or absence of fouling therein. For example, when turbidity probes are used to estimate nucleation induction time $[9,10]$, the method is unable to distinguish appearance of crystals in the liquid phase from that on vessel walls if and when fouling occurs.

Imaging is another technique commonly used to track various properties of multiphase systems containing suspended solid particles and/or bubbles [11], identify nucleation induction time [12, 13] or crystal growth [14] in industrial settings. A review of recent advances in monitoring and control of crystallisation systems using imaging can be found in [15]. Use of in-situ endoscopy-stroboscopy [16] equipment and multivariate image analysis for process monitoring has been demonstrated. Estimating nucleation induction time in the bulk through Bulk Video Imaging [17] using multivariate image analysis and by converting pixel intensities time series of acoustic signals allows to eliminate stirrer effects through band-stop filtering in batch crystallisers. Methods to automatically estimate induction time using Shewhart Charts was shown in $[17,18]$. These techniques apply to bulk monitoring only and to our knowledge there is no previously published method for detection 
of fouling through image analysis.

Fouling in an important problem in both batch and continuous crystallisation. It is especially significant for continuous crystallisation processes where early detection of fouling can be used to guide relevant corrective actions in order to keep process running. In this work a batch system has been designed which locally mimics the behaviour of a continuous plug-flow type crystallisation platform, in terms of keeping the local temperature and solution concentration constant, at least until the appearance of first crystals at walls or in the bulk. The approach proposed here is applicable subject to visual access to the crystalliser walls and uses an off-the-shelf commodity camera, pointing towards an area of interest at solid-liquid interface while looking through a transparent crystalliser wall ${ }^{1}$. Statistical analysis of the acquired images allows distinguishing crystal appearance in the bulk solution from fouling on the solid surfaces. Note that the induction times determined here refer to the location where crystals are observed and appearance of crystals at that location does not necessarily mean that nucleation occurred there. The aim of the proposed technique is to separately determine induction times for appearance of crystals at solid surfaces and in the bulk automatically and in real-time, by providing a sensitive early warning system for detection of fouling in crystallisation processes.

\footnotetext{
${ }^{1}$ Industrial settings with opaque walls will require appropriate adjustments.
} 


\section{Methodology}

\subsection{Experimental Setup}

The experimental setup used to monitor fouling at glass crystalliser walls under isothermal conditions was a batch system known as a moving fluid oscillatory baffled crystalliser (MFOBC). However, we note that the imagebased monitoring and image analysis approach proposed here can be used to analyse data collected from any other experimental setup where real-time images of vessel walls are available, under batch or continuous, isothermal or non-isothermal conditions.

The MFOBC has orifice baffles spaced equally throughout the glass pipe which are overlaid with the direction of fluid oscillation [19]. Local mixing is facilitated through Eddie formation due to the interaction of the baffles with the oscillatory flow. The oscillation conditions are defined through frequency and amplitude [20]. This system can achieve close to plug flow when operating under continuous conditions, as a continuous oscillatory baffled crystalliser (COBC), at relatively low net flow rates while good heat and mass transfer is radial direction as well as solid suspension is facilitated by oscillatory motions. In a typical COBC setup there are several glass sections operated under different temperatures and fouling is likely to occur in a section with highest supersaturation. In order to monitor the behaviour in such a section independent from the rest of the COBC setup, a physical batch model locally representing the COBC conditions was constructed by removing the section of interest from the continuous arrangement and mounting it vertically between two other sections providing suitable boundary conditions to prevent nucleation outside of the section of interest by keeping the 


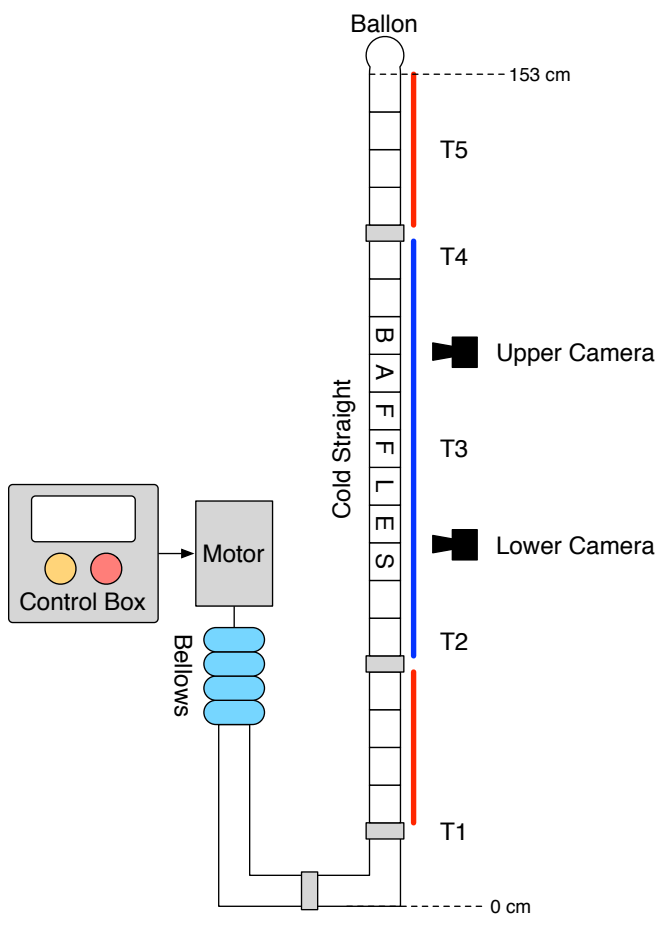

Figure 1: Experimental setup showing positions of temperature measurement, cameras, glass sections and collars which connect the glass components together.

temperature of the fluid outside of the section above saturation temperature. The resulting batch setup can reproduce local temperature, concentration and flow conditions identical to the relevant section of the COBC under corresponding conditions, in the absence of any solid phase until onset of crystal formation is observed.

The MFOBC used here (shown in Figure 1) consists of tubular jacketed glass sections with orifice baffles spaced equally throughout. Each full glass sections is made up of 22 individual cells of volume $5 \mathrm{ml}$ and internal diameter $15 \mathrm{~mm}$ (DN15). The batch setup contains one half glass straight attached to bellows and kept at temperature T1, followed by one full straight where 
desired target temperature is kept at the position of monitoring cameras to achieve required supersaturation, and then another half straight at the top kept at temperature T5, completing the setup. The principle behind this configuration is that while all three regions are interconnected, the temperatures are calibrated so that the cameras are always monitoring regions of fixed temperature. The two half straights above and below are kept hot to prevent seeding from above and below, respectively. Temperatures T1 and T2 are monitored during experiments via built-in thermocouples. Temperatures T3, T4 and T5 are recorded for calibration purposes prior to the experiment as multiple thermocouples in the system may affect the fouling process.

Two sets of oscillations conditions were used for fouling experiments, high oscillations with frequency and amplitude of $2 \mathrm{~Hz}$ and $45 \mathrm{~mm}$, respectively, and low oscillations with frequency and amplitude of $1 \mathrm{~Hz}$ and $45 \mathrm{~mm}$, respectively. Table 1 gives temperature information pertaining to the two oscillation conditions used for the experiments.

L-Glutamic Acid (LGA) was crystallised from water in this setup. The supersaturations of the solutions were calculated from the solubility at $20^{\circ} \mathrm{C}$, which has a value of $7.07 \mathrm{~g} / \mathrm{L}$ of water [21, 22]. Supersaturations 3, 4 and 6 were used. Hot solution at $20^{\circ} \mathrm{C}$ was prepared out with the MFOBC and pumped while hot in to the MFOBC using a peristaltic pump via a port at the base of the equipment. This was filled so that the upper half section was $75 \%$ full. At this point the oscillation was set and the experiment was run and images were taken. Two Microsoft LifeCam VX-3000 cameras are used and LED torch is used for illumination inside an enclosed environment to ensure constant lighting and to minimise reflections. The jacket temperatures of the 


\begin{tabular}{|l|c|c|}
\hline Position on MFOBC & High Oscillation & Low Oscillation \\
\hline T1 $(16.5 \mathrm{~cm})$ & $82^{\circ} \mathrm{C}$ & $84.0^{\circ} \mathrm{C}$ \\
\hline T2 $(54.5 \mathrm{~cm})$ & $26.8^{\circ} \mathrm{C}$ & $29.5^{\circ} \mathrm{C}$ \\
\hline Lower Camera $(66.5 \mathrm{~cm})$ & $22.0^{\circ} \mathrm{C}$ & $23.3^{\circ} \mathrm{C}$ \\
\hline T3 $(85.5 \mathrm{~cm})$ & $19.5^{\circ} \mathrm{C}$ & $19.8^{\circ} \mathrm{C}$ \\
\hline Upper Camera $(88.0 \mathrm{~cm})$ & $19.5^{\circ} \mathrm{C}$ & $19.7^{\circ} \mathrm{C}$ \\
\hline T4 $(110 \mathrm{~cm})$ & $23.7^{\circ} \mathrm{C}$ & $24.6^{\circ} \mathrm{C}$ \\
\hline T5 $(134 \mathrm{~cm})$ & $38.4^{\circ} \mathrm{C}$ & $41.5^{\circ} \mathrm{C}$ \\
\hline
\end{tabular}

Table 1: Temperature Profiles for High Oscillation Conditions (2 Hz, $45 \mathrm{~mm}$ ) and Low Oscillation Conditions (1 Hz, $45 \mathrm{~mm})$.

glass straights were preset before being filled with hot solutions in order to quickly achieve the desired bulk solution temperatures. A balloon was placed over the top section of the MFOBC to eliminate effects from evaporation.

\subsection{Image Preprocessing}

The web camera output is a series of JPEG images with resolution of 320x240 pixels. Each pixel consists of a tuple of the Red, Green and Blue 8-bit colour components. The 8-bit component values allow colour intensities to be captured in the range of 0 and 255 , where 0 is no presence and 255 is maximum presence of the corresponding component. A pixel with value $(\mathrm{R}, \mathrm{G}, \mathrm{B})=(255,255,255)$ represents a white pixel, while a pixel with value $(\mathrm{R}, \mathrm{G}, \mathrm{B})=(0,0,0)$ represents a black pixel.

The sequence of RGB JPEG images is converted to grey scale images using the formula $0.2989 \mathrm{R}+0.5870 \mathrm{G}+0.1140 \mathrm{~B}$ which is the well accepted NTSC 


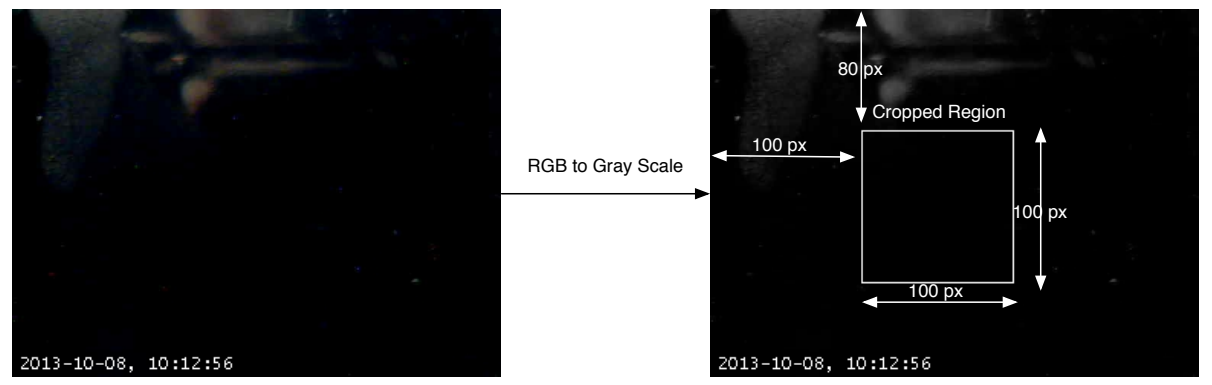

Figure 2: Image pre-processing.

standard for luminance. The images are cropped to 100x100 pixels, with offset of 80 pixels from the top and 100 pixels from the left (shown in Figure 2), in order to eliminate reflections from the cylindrical reactor walls. The exact location of cropping is not important as long as reflections due to the pipe curvature and the time stamp annotations are eliminated. As a result, the initial cropped frame has only black pixels (intensity 0) when the crystalliser has no growth in the bulk or walls. The cropping is performed in all images acquired and the resulting image sequence consists of a 3 dimensional array with $N=100$ rows by $M=100$ columns and $K$ frames. The instantaneous intensity of a pixel at location $(i, j)$ at time $t$ is denoted by $I_{i j}(t)$.

\subsection{Analysis Methodology}

Often experimentalists capture signal traces (profiles) and estimate induction time offline after the experiments are finished using a first order polynomial regression near the region of the crystal appearance. This kind of approach clearly does not allow early detection of crystal appearance and/or fouling events since an onset of change in relevant measurable quantities can only be determined after the extent of change becomes very significant. 
Moreover, choice of regression window can be arbitrary and often cannot be uniquely determined. An online real-time method to identify induction time is usually based on selection of a predetermined, fixed amplitude threshold and when the signal traces exceed that threshold, it is concluded that the induction time has been observed. Although this may be adequate for well characterised setups with known compounds and experimental conditions, it is impractical when applied to new systems or under different conditions or equipment. In order to address this challenge, a Bayesian Online Change Point Detection technique is presented in Section 2.4 for the automatic estimation of the induction time independent of setup, compound or conditions and it is agnostic of the signal trace used (i.e. sensor type). Its applicability for a wide range of signal traces is demonstrated throughout this work as it is the method chosen here to identify induction time in either bulk solution, at solid surfaces, or both combined.

Typical signal traces used to measure induction times are provided by Process Analytical Technology (PAT) equipment based on spectroscopic, scattering or video/imaging methods. However, such equipment is generally only able to detect induction time for crystal appearance in the bulk solution and in case signals from both bulk solution and vessel walls are recorded, it provides information on combined bulk and wall changes together. Some instruments such as the Mettler Toledo FBRM now provide a fouling index indicator for the probe window however these may not be representative of the encrustation levels experienced on the reactor walls, since probe coating materials are often different from those on the reactor walls hence varying the degree of encrustation. Some previous reports in the literature have em- 


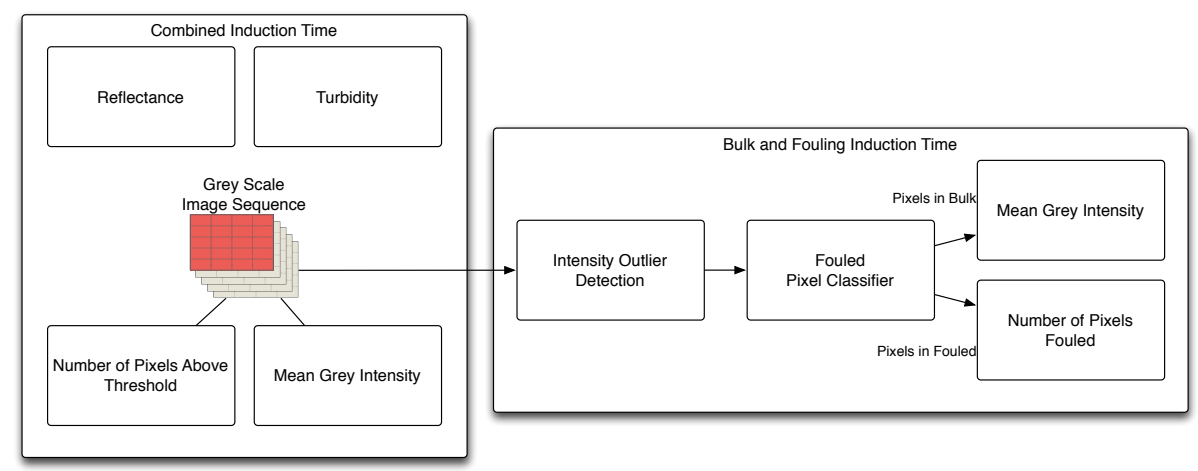

Figure 3: Signals that can be used to estimate combined induction time and the proposed method to separate crystal appearance in the bulk solution and at the solid surfaces.

ployed imaging systems to estimate induction time $[12,13]$. These techniques are able to report in real-time the induction time using Mean Grey Intensity as a signal trace and amplitude thresholding as a detection method. Their performance is similar to the that of PAT equipment such as ATR-IR [13]. Another rudimentary signal trace is to count the number of pixels above an intensity threshold. All the signal traces that can be used to measure combined induction time are illustrated in Figure 3 and described in detail in Section 3.

Figure 3 shows the proposed approach to detect separately crystal appearance in the bulk solution and on the vessel walls. The acquired images are initially passed through an Intensity Outlier Detector which highlights pixels with higher intensity. These pixels are subsequently processed by the Fouled Pixel Classifier that infers pixel state as fouled and non-fouled. The non-fouled pixels (i.e. in the bulk) are subsequently processed to generate the Mean Grey Intensity signal trace for estimation of the bulk induction time, while the fouled pixels are counted to provide a fouling index indicator 
and estimate the fouling induction time. The bulk and fouling induction time estimations are described in detail in Section 4.

\subsection{Automatic Change Point Detection}

A typical progression profile from turbidity, reflectance or image Mean Grey Intensity start with low amplitude and as crystals appear, the amplitude rises. Induction times can be determined in a post-processing fashion after an experiment is completed or online, through thresholding the amplitude of the signal trace. Although these techniques are effective, they are sensitive to acquisition equipment and experimental conditions with parameter tuning necessary. In order to overcome these limitation, a Bayesian Online Change Point Detection [23] approach is used here, modelling the data as a Poisson process and the rate of exponential prior on the change point interval $\lambda_{g a p}=$ 1000 (i.e. change point distribution) in a similar fashion to the Coal Mine Disaster Data in [23].

The top plot in Figure 4 shows the MGI data of an experiment against time. For every new observation (i.e. MGI value), while the parameter $\eta$ of the Poisson distribution remains the same, there is no occurrence of a change point and the run length increases by 1 . When a new observation appears that comes from a Poisson with a different parameter $\eta^{\prime}$, the run time drops indicating a change point. The change point interval has rate $\lambda_{\text {gap }}$ which models probabilistically the occurrence of a change point.

At the bottom graph of Figure 4 the intensity plot shows the posterior probability of the current run length $P\left(r_{t} \mid x_{1: t}\right)$ at each time step $t$ using a logarithmic scale. Darker pixels indicate higher probability while white pixels indicate zero probability. For a time step $t$, a column of pixels of the intensity 

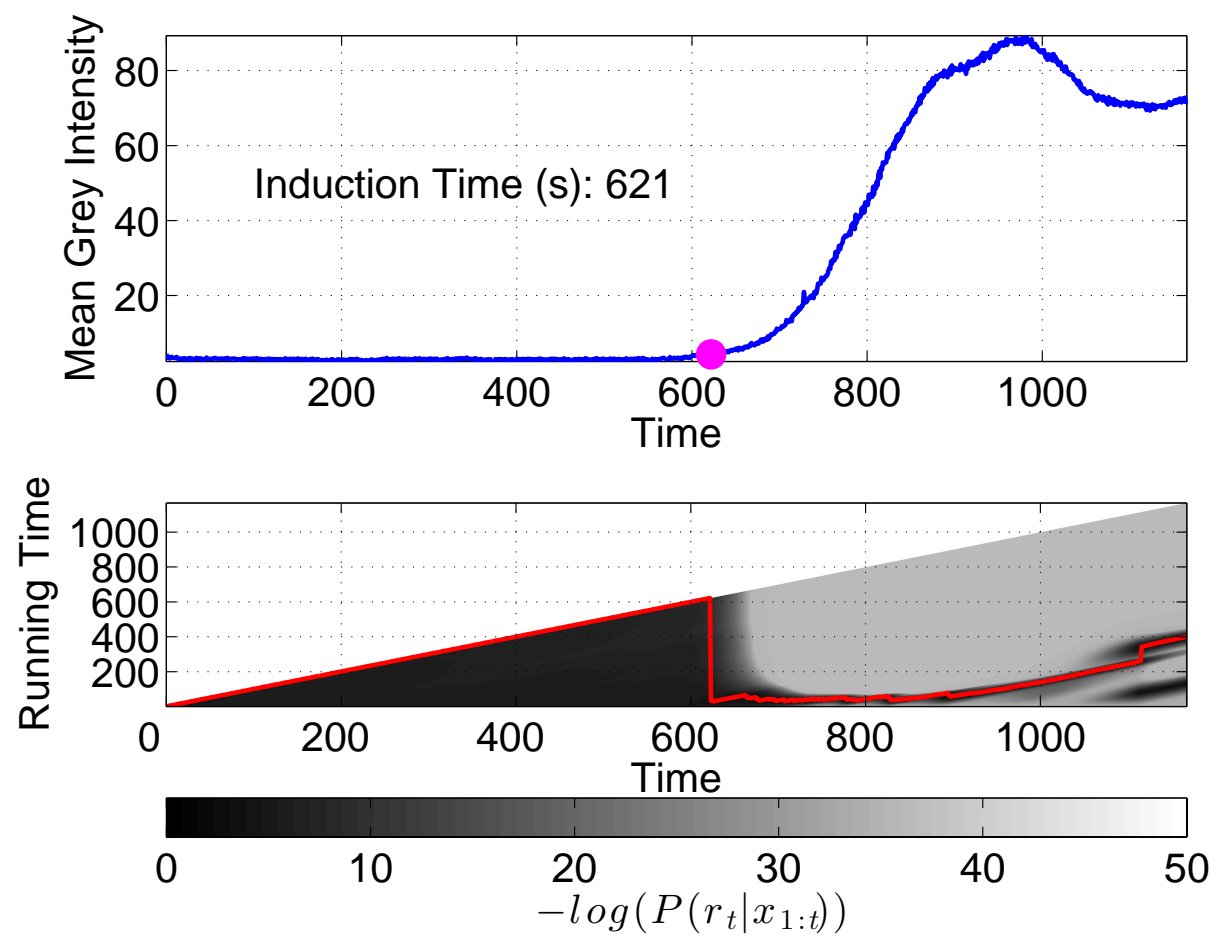

Figure 4: Top: Mean Grey Intensity profile against time. Bottom: The intensity plot shows the probability of the current run time $P\left(r_{t} \mid x_{1: t}\right)$ at each time step (in logarithmic scale). The most probable run time $r_{t}$ is annotated with the red line.

plot illustrate the probability mass function for every run time/length.

For example, for $t=600$, the run time above 600 has zero probability and hence the $-\log (0)=\operatorname{Inf}$ which is shown as white. For run time below 600 , the probability is between 0 and 1 which results in a shade of grey. The highest probability occurs at run time 600 which is the darkest pixel on that column and a change point has yet to occur. Similarly, for time $t=1000$, the maximum occurs at run length 140 (a number of changes points have already occurred). The most probable run times are annotated with a red line on the bottom graph of Figure 4. 
The algorithm finds the negative slopes on the most probable run times (i.e. the points where the run time is reset) and these are the detected change points. The first change point is attributed to the onset of crystal formation. Subsequent change points are caused from breakage of encrusted regions and re-encrustation, but those are not considered in this analysis.

\section{Combined Induction Time Estimation}

In this section, the Bayesian Online Change Point Detection algorithm is applied in a number of signal traces to estimate combined induction time. The algorithm is applied to turbidity, FBRM and image based signals as shown in Figure 3.

As an example, consider a crystallisation experiment for LGA with concentration $45 \mathrm{~g} / \mathrm{L}$ in water, oscillation amplitude of $30 \mathrm{~mm}$ and frequency $1.5 \mathrm{~Hz}$. The measured profiles of the experimental signals are shown in Figure 5. The induction times based on the algorithm presented in Section 2.4 using the FBRM and turbidity signal traces are 23.50 and 27.25 minutes respectively.

Previous applications of image processing to estimate induction time have proposed an automatic detection from Mean Grey Intensity (MGI) signal traces $[12,13]$. These works have identified that the size of the interrogation window affects the induction time estimation. Large windows spatially average a greater area and consequently, might miss the early appearance of crystals. On the counter side, small windows are prone to noise and other effects such as bubbles may lead to false detections.

Formally, the mean grey intensity $\bar{I}(t)$ for every frame at time $t \in\{1,2, \ldots, K\}$, 


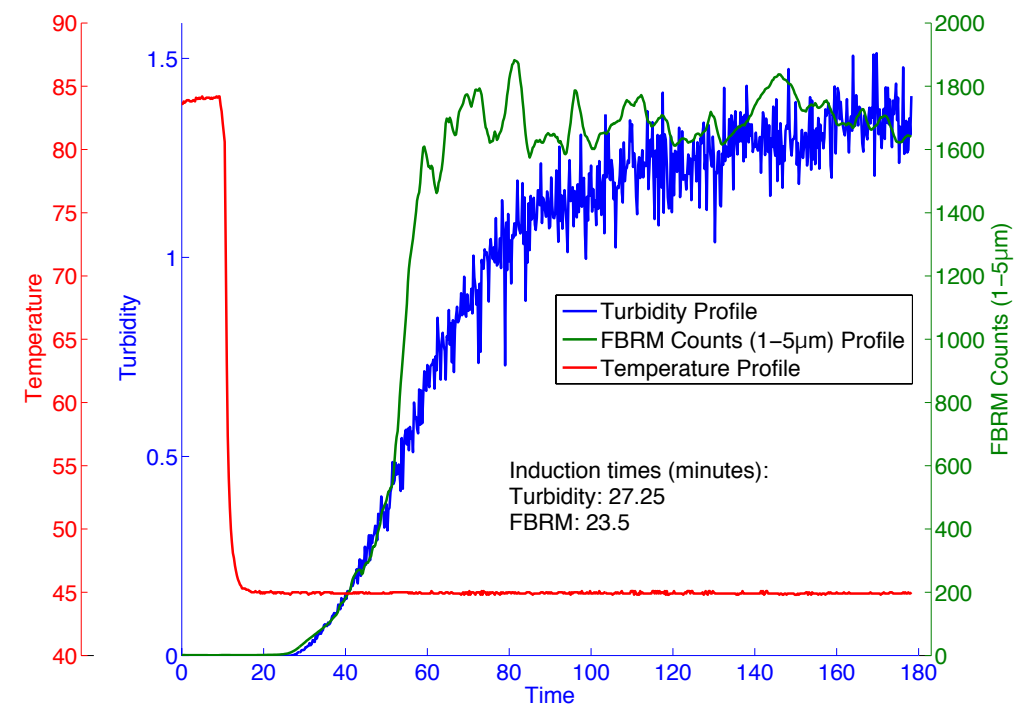

Figure 5: Temperature, Turbidity and FBRM signal traces for crystallisation of LGA in water (experiment with no fouling).

as:

$$
\bar{I}(t)=\frac{\sum_{i=1}^{N} \sum_{j=1}^{M} I_{i j}(t)}{N M}
$$

where $I_{i j}(t)$ is the instantaneous grey intensity of the pixel $(i, j)$ at time $t$.

Often, the limitations of MGI traces is that it dampens the early detection of crystals that flow through the camera view finder making the method of estimating induction time through MGI is sensitive to the interrogation window size. The Mean Grey Intensity curves against time for various interrogation window sizes are shown in Figure 6. The estimated induction time for all interrogation windows is 25.75 minutes and the effect of the win- 
dow sizes is not significant. Unlike the work presented in [12] the camera resolution used here is low and individual crystals can not be detected with confidence hence some sensitivity is lost. Also, the proposed change point detection algorithm estimates the induction time in a probabilistic fashion and even if there are some spikes in the waveform. For example for windows 10x10 px and 20x20 px, the waveforms have spikes before the detected induction point which was most likely caused from bubbles passing through the window.

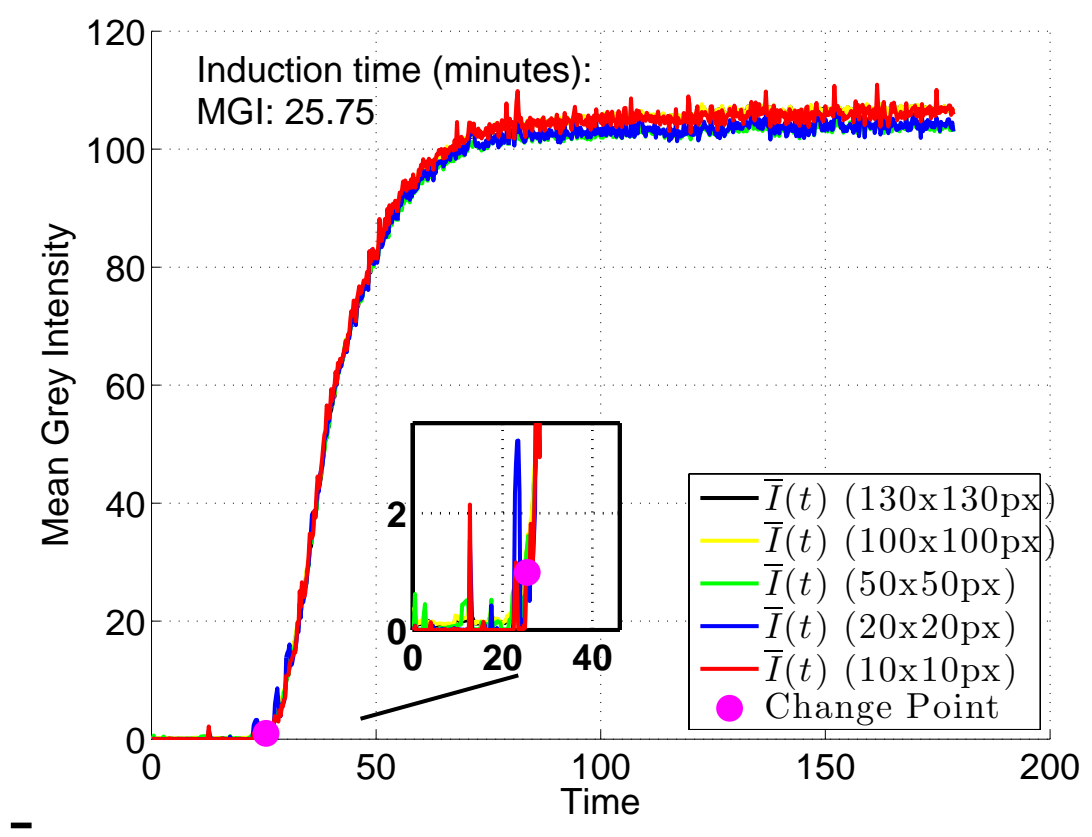

Figure 6: Mean Grey Intensity profile for various interrogation window sizes. LGA in water (experiment with no fouling).

Another method which could increase sensitivity compared to MGI is counting pixels above a threshold. Intuitively, the crystal appearance has started when the solution in the crystalliser gets cloudy i.e. the intensity of 
the pixels in the frame start to rise. Frequently experimentalists capture images at regular intervals to retrospectively verify the experiments. A standard method to determine process progression is to select an intensity threshold $\tau$ and at every time instance $t \in\{1,2, \ldots, K\}$, count the percentage of pixels exceeding the threshold ${ }^{2}$. More formally for a threshold $\tau \in\{1,2, \ldots, 255\}$, the function counting the ratio of pixels above the threshold is:

$$
C_{\tau}(t)=\frac{\sum_{i=1}^{N} \sum_{j=1}^{M} u_{\tau}\left(I_{i j}(t)\right)}{N M}
$$

where $u_{\tau}(x)$ is the step function:

$$
u_{\tau}(x)= \begin{cases}1 & \text { if } x \geq \tau \\ 0 & \text { if } x<\tau\end{cases}
$$

Figure 7a shows the percentage of pixels above the threshold $\tau$ versus time. Applying the change point detection method on the threshold signal traces, indicates that the estimated induction varies significantly with the threshold value $\tau$. The relation between $\tau$ and the estimated induction time is shown in Figure 7b. The induction time increases almost linearly with the threshold. Low intensity thresholds provide high sensitivity however, application of this technique on other datasets with higher image noise, did not provide a robust estimation. Low thresholds produce to spiky signal traces, especially during the beginning of the experiment where camera noise is significant leading to incorrect estimation of induction time. For high

\footnotetext{
${ }^{2}$ Note: This threshold is applied on all pixels of every frame in the image sequence, unlike the MGI comparison threshold mentioned previously for detection purposes.
} 


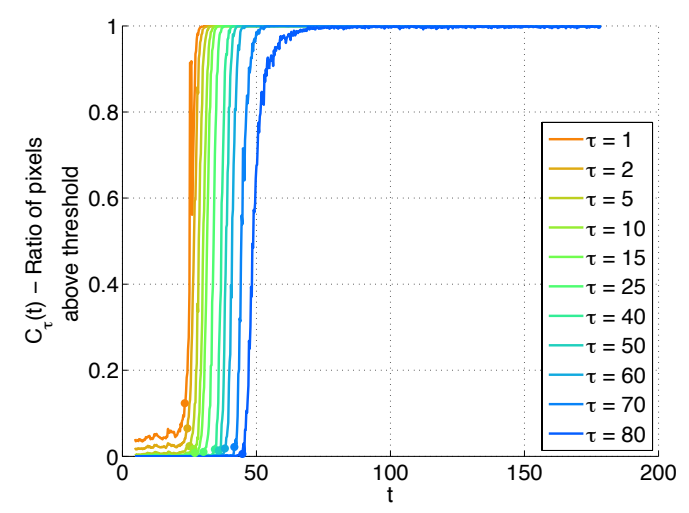

(a) a waveform rise.

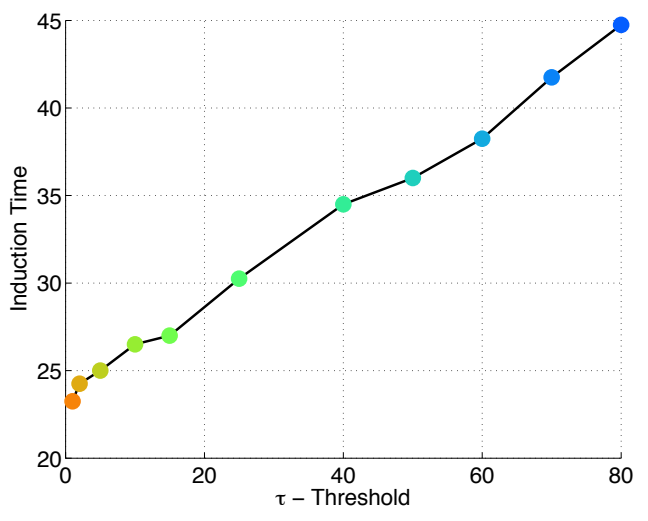

(b)

Figure 7: LGA in water (experiment with no fouling): (a) Percentage of pixels above threshold $\tau$ versus time for various threshold levels, (b) Induction time for varying threshold levels.

thresholds, the crystal appearance must be well developed in order to observe

The combined induction times obtained from various signal traces when the Bayesian Online Change Point Detection algorithm is applied are summarised in Table 2. FBRM signal traces are the most sensitive followed by the MGI and Turbidity.

The turbidity probe has no means to detect fouling and hence measure the combined induction time. The FBRM probe is able to distinguish encrustation on the probe through fouling index however, does not capture any information related to fouling on the crystalliser walls.

Estimating induction time through imaging contain information related to the fouling of the crystalliser walls, however this information is not being utilised. 


\begin{tabular}{|c|c|}
\hline Parameter & Induction Time (minutes) \\
\hline FBRM & 23.50 \\
\hline MGI & 25.75 \\
\hline Turbidity & 27.25 \\
\hline Pixel Thresholding & $(23.25-44.75)$ Varying with threshold $\tau$ \\
\hline
\end{tabular}

Table 2: Comparison of induction time from various signal traces. LGA in water, (experiment with no fouling).

\section{Fouling and Bulk Induction Time}

Images from a commodity web camera pointing towards the crystalliser walls does not only contain information about crystal appearance in the bulk but also the crystalliser walls. This section presents a method to separate fouling and bulk induction time through statistical analysis of the acquired images as summarised in Figure 3.

Crystals moving through the camera view result in variations in pixel intensity. Regions where crystals are present will have higher pixel intensity compared to background regions. Crystals stuck on the crystalliser walls are closer to the camera viewfinder and reflect more light. Crystals in the bulk also reflect light leading to high pixel intensities, however the pixel intensities are not consistently high for a consecutive number of frames. As the crystals move away with the liquid flow intensities drop. The proposed pixel detection algorithm consists of two steps:

1. Identify pixels with the highest intensity.

2. Identify pixels which have the highest intensity for a consecutive number of frames. This rule in necessary to avoid false positives where 
particles larger/brighter than the encrusted region are passing through the view.

To achieve the first step of the process an upper outlier detection method based on Chebyshev's inequality is used [24]. The inequality provides a bound on the percentage of data point falling further than $k$ standard deviations away from the mean. On this occasion the inequality is applied to the pixel intensities distribution of the frame at time $t$.

$$
P(|I(t)-\bar{I}(t)| \geq k \sigma) \leq \frac{1}{k^{2}}
$$

The inequality is used to determine upper and lower Outlier Detection Value $(\mathrm{ODV})$ limits $\left(O D V_{U}\right.$ and $\left.O D V_{L}\right)$ and does not make any assumptions on the underlying data distribution. Pixels with intensity outside the limits, are classified as outliers. For $k=5$ this leads to maximum $4 \%$ of pixels been classified as outliers on both directions. The outlier detection limits are given by:

$$
\begin{aligned}
O D V_{L} & =\bar{I}(t)-k \sigma \\
O D V_{U} & =\bar{I}(t)+k \sigma
\end{aligned}
$$

From these two detection values, only upper outliers (greater than $O D V_{U}$ ) indicate fouled regions and the following detection function is defined:

$$
d_{i j}(t)= \begin{cases}I_{i j} & \text { if } I_{i j} \geq O D V_{U} \\ -I n f & \text { otherwise }\end{cases}
$$




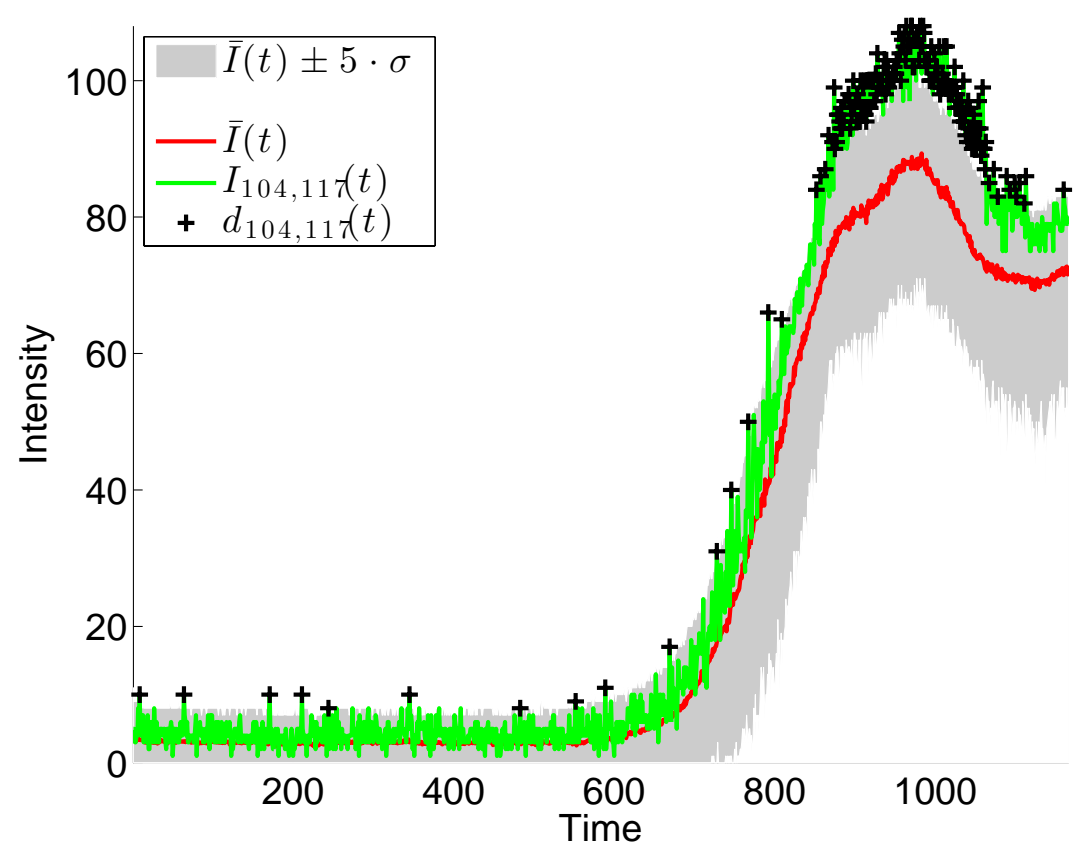

Figure 8: Mean Grey Intensity profile with Chebyshev's outlier detection bounds for $k=5$. The green line shows the instantaneous intensity of pixel $(104,117)$, while the black crosses indicate the points where the pixel intensity has exceeded the $O D V_{U}$; i.e. the output of the $d_{i j}(t)$ from Equation 6. LGA in water (experiment with fouling).

The detection function has value of - Inf for pixels that are not detected as outliers while the detection function is equal to the corresponding pixel intensity $\left(I_{i j}\right)$.

Figure 8 shows in red line the MGI profile curve, while the grey shaded area shows the intensities that lie between the Outlier Detection Value Lower $\left(O D V_{L}\right)$ and the Outlier Detection Value Upper $\left(O D V_{U}\right)$ from Eq. 5. The green line shows the instantaneous pixel intensity of an arbitrary pixel at location $(104,117)$. Every time pixel intensity exceed the $O D V_{U}$, the pixel is classified as an outlier as in Eq. 6 and is annotated on the graph with black 
crosses. All pixels that have intensity lower than the upper bound $O D V_{U}$ have detection value of $-\operatorname{Inf}$. Not all pixels that the detection function has identified as outliers, are necessarily fouled. The high intensity can be caused either due to camera noise or objects passing through that pixel region. However, pixels that the detection function has consistently identify as outliers; i.e. for $N_{S}$ consecutive number of frames, are fouled and the following filtering algorithm is used to detect those:

1. For time step $t$, create a set $\mathcal{A}(t)$ with all pixels in the frame

$$
\mathcal{A}(t):\{(i, j)\} \text {, for all } i \in\{1, \ldots, N\} \text { and } j \in\{1, \ldots, M\}
$$

2. Using the set $\mathcal{A}(t)$ estimate the $O D V_{U}$ required for the outlier detection function in Eq. 6.

3. Create a set $\mathcal{F}(t)$ with all the pixels that their $d_{i j}$ is greater than or equal to 0 for the previous $N_{S}=5$ time steps/frames. Essentially, this steps looks to previous images to determine if the pixels where consistently an outlier based on the instantaneous detection function.

$$
\begin{array}{r}
\mathcal{F}(t):\{(i, j)\}, \text { where } d_{i j}(t-l) \geq 0, \\
\text { for all } l \in\left\{1, \ldots, N_{S}\right\}, i \in\{1, \ldots, N\}, j \in\{1, \ldots, M\}
\end{array}
$$

4. The number of fouled pixels are equal to cardinality (number of elements) of the set $\mathcal{F}(t):|\mathcal{F}(t)|$.

5. Recompute $O D V_{U}$ for the set of pixels in the intersection $\mathcal{A}(t) \cap \overline{\mathcal{F}}(t)$ (i.e. exclude pixels that were classed as fouled in the current time $t$ ).

6. Go to step 3 . 

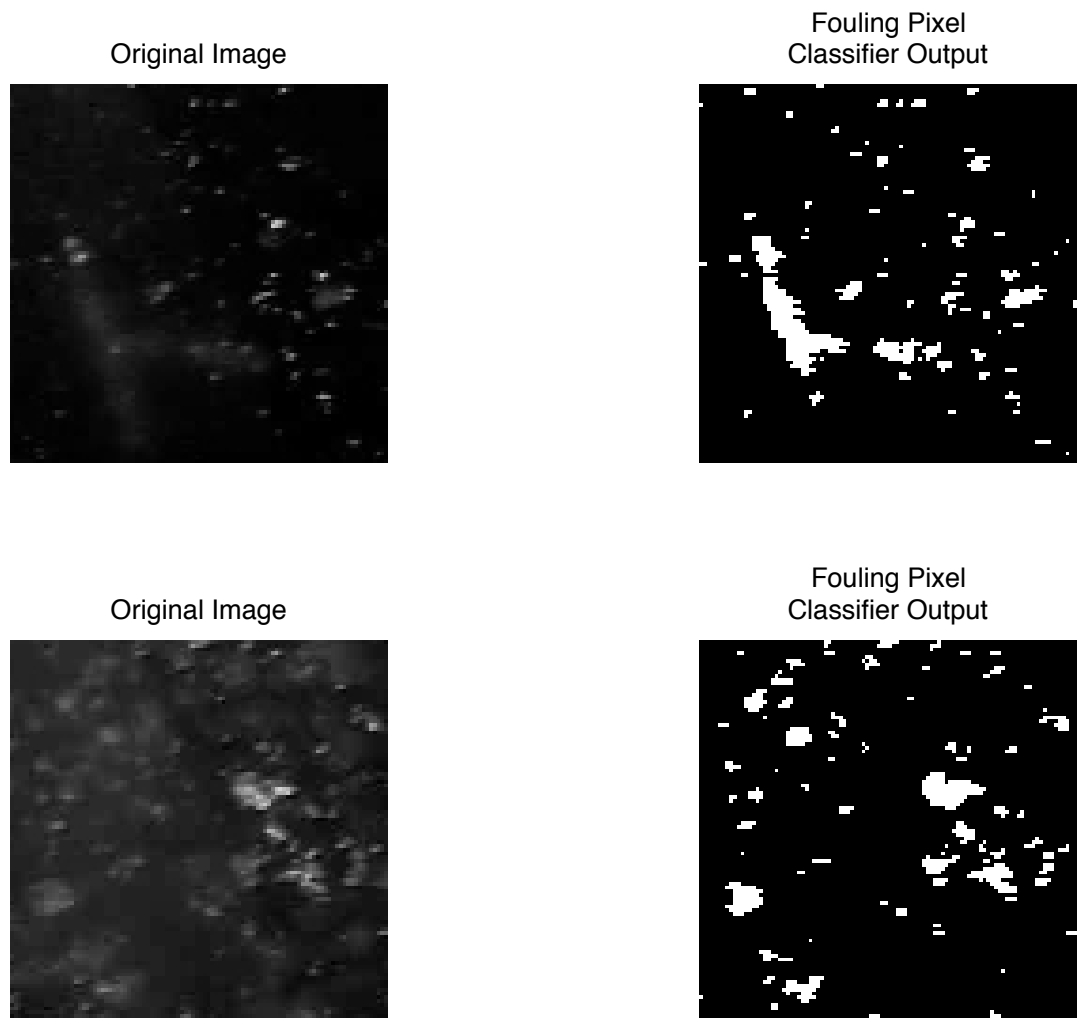

Figure 9: Two examples of original images and outputs of the fouling classifier. LGA in water (experiment with fouling).

The output of the classification algorithm can subsequently be used to visualise regions where fouling has occurred. Figure 9 shows two example images and their corresponding outputs of the classifier.

Figure 10 shows the percentage of pixels classified as fouled, against time which is obtained as the ratio of the cardinality of the set $\mathcal{F}(t)$ over the total number of pixels in the frame. The percentage of pixel can be used as a fouling index indicator at any stage of the experiment. Figure 10 also shows the combined MGI signal trace i.e. without taking in consideration the pixel 


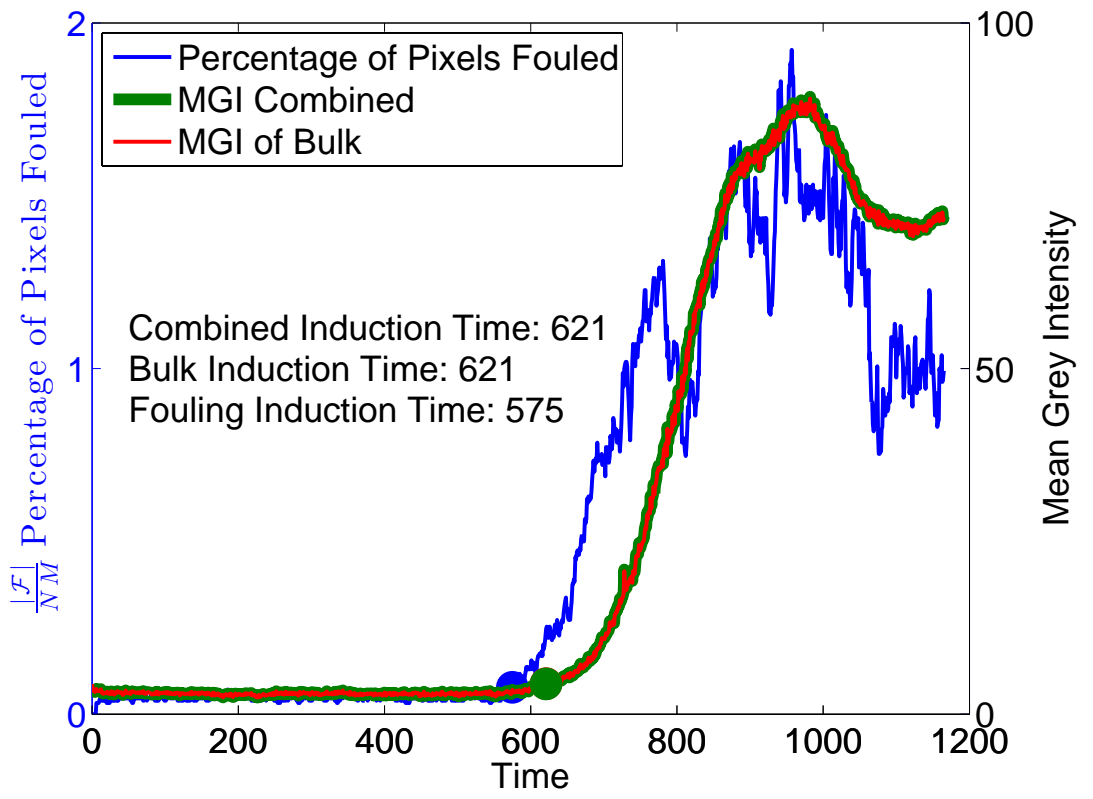

Figure 10: Signal trace profiles for fouling index, bulk MGI and combined MGI. LGA in water (experiment with fouling).

class as in Section 3, while the MGI bulk signal trace only considers pixels that are not fouled (i.e. belonging to the bulk).

In this experiment, the combined MGI and Bulk MGI are almost identical and this is due to a low number of fouled pixels (i.e. only $2 \%$ of pixels are fouled). Using the change point detection to estimate induction times from these signal traces, fouling appears at 575 seconds, while induction in the bulk occurs at 621 seconds, with identical time for the combined trace.

It is important to highlight that the fouling induction time is detected before bulk or combined induction times. This is justified when considering that the fouling signal trace provides higher sensitivity when compared to MGI traces. The MGI traces inherently damp localised changes until the 


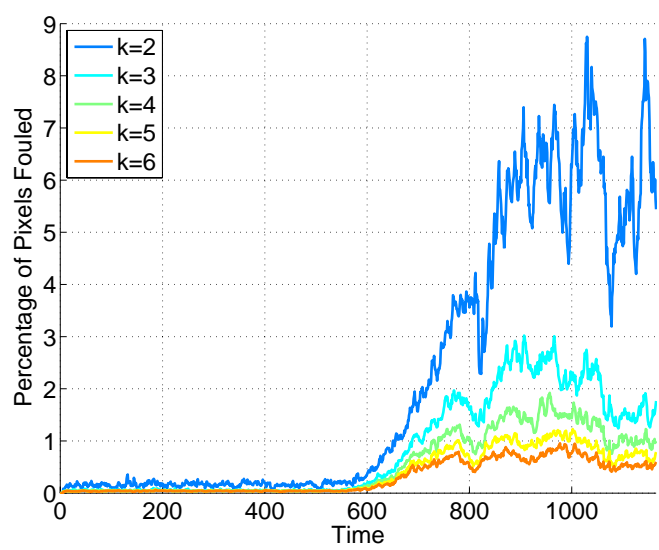

(a)

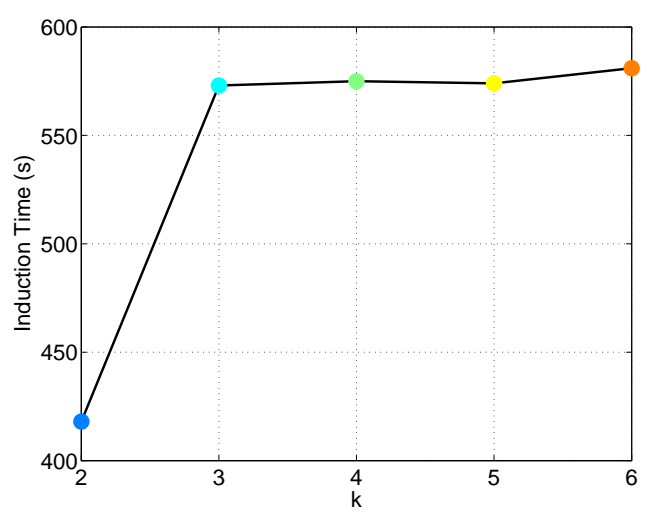

(b)

Figure 11: (a) Percentage of fouled pixels versus time for various $k$, (b) Induction time against $k$.

intensity of a significant number of pixels has increased. This difference in sensitivity introduces a potential uncertainty of the order of events, e.g., when induction times on the wall and in the bulk are near each other. The aim in this work is to provide an early warning system for fouling and this is achieved through the high sensitivity provided by the fouling signal trace. Measuring bulk induction time with high sensitivity could be achieved through the use of other PAT equipment such as reflectance (i.e. FBRM).

\subsection{Sensitivity of Fouling Classifier Parameters}

The fouling pixel classifier sensitivity can be adjusted through two parameters. The outlier detection sensitivity $k$ standard deviations and the number of consecutive frames $N_{S}$ a pixel has to remain an outlier, before it is classified fouled.

Figure 11a shows the percentage of pixels against time for various $k$ stan- 
dard deviations and $N_{S}=5$. The maximum percentage of pixels intensities beyond $k=2$ standard deviations from the mean is $25 \%$ hence a greater number of pixels have the potential to be identified from the detection function in Eq. 6. Higher values of $k$ restrict the bound and hence sensitivity of the detection function. For $k=6$ the maximum percentage of pixels is restricted to $2.77 \%$. The fouling induction time against $k$ is shown in Figure 11b. For $k=2$ the bound is wide and pixels in the bulk are classified prematurely as fouled. However, as the $k$ increases to 3 and beyond the sensitivity reduces resulting to identical induction times.

Similar sensitivity analysis is performed for $N_{S}$; the number of consecutive frames a pixel has to be identified an outlier for a fixed value of $k=5$. The percentage of fouled pixels against time are shown in Figure 12a. The longer the time period a pixel remains an outlier the more sever the fouling. As expected, the lower the $N_{S}$ greater chances that the pixel intensity is an outlier and consequently highlighted as fouling. As the $N_{S}$ increases pixels have to remain outliers for a longer period of time. The fouling induction time against $N_{S}$ is shown in Figure 12b. It should be noted that although the algorithm is sensitive to the selection of the $N_{S}$ parameter, the relative change in induction time is less the $5 \%$ of the absolute induction time. Inspection of the acquired images indicate that parameters $k=5$ and $N_{S}=5$ are the most suitable to detect fouled pixels regions.

\section{Conclusions}

In this article we presented a method to automatically detect induction time through Bayesian Online Change Point Detection in real-time while the 


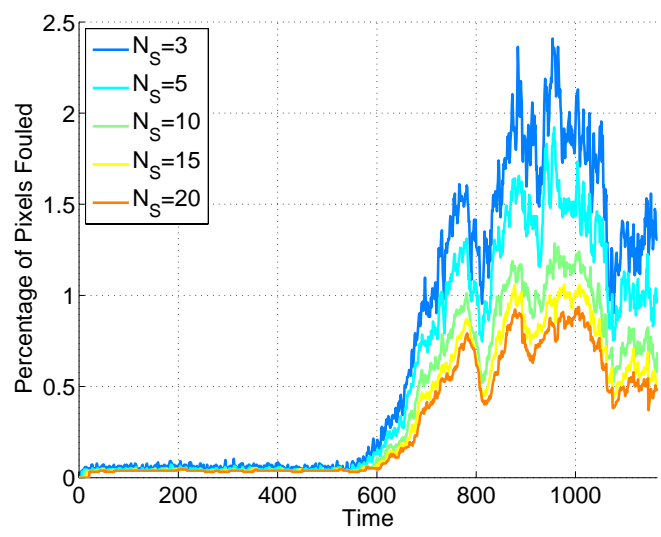

(a)

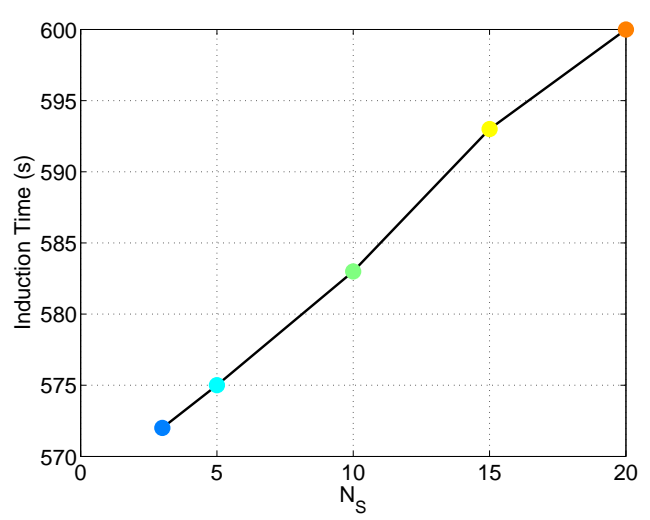

(b)

Figure 12: (a) Percentage of fouled pixels versus time for various $N_{S}$, (b) Induction time against $N_{S}$.

experiment is in progress. It is demonstrated that the change point detection algorithm can estimate induction time for various types signal traces such as reflectance, turbidity, Mean Grey Intensity and percentage of pixels fouled without the need to tune model parameters. This approach to estimating induction time is more robust than thresholding where the estimates can vary significantly with the threshold selection.

We have also developed a novel method for the early detection of fouling through commodity web cameras. Classification of image pixels corresponding to either bulk solution or fouled surface was achieved through statistical analysis of pixel intensity time series. The proposed technique is applicable to industrial settings were visual access to the fouling surface is available.

The number of pixels classified as fouled is an indicator of the degree of fouling at every stage of the experiment. Using the fouling indicator and applying the automatic change point detection, fouling induction time can 
be estimated. We note that surface fouling and bulk crystallisation signal traces provide significantly different sensitivity and that may introduce an uncertainty in the order of events when induction times at surface and in the bulk are close to each other.

\section{Acknowledgements}

The authors would like to acknowledge financial support from the EPSRC, AstraZeneca and GSK. This work was performed as part of the "Intelligent Decision Support and Control Technologies for Continuous Manufacturing and Crystallisation of Pharmaceuticals and Fine Chemicals" (ICT-CMAC) Project (grant number EP/K014250/1).

\section{References}

[1] T. Bott, Aspects of crystallization fouling, 1997. doi:10.1016/S08941777(96)00137-9.

[2] J. W. Mullin, Crystallization, 4th ed., Butterworth Heinemann, 2001.

[3] T. Geddert, W. Augustin, S. Scholl, Induction Time in Crystallization Fouling on Heat Transfer Surfaces, Chemical Engineering \& Technology 34 (2011) 1303-1310.

[4] E. Wallhäuß er, M. Hussein, T. Becker, Detection methods of fouling in heat exchangers in the food industry, Food Control 27 (2012) 1-10.

[5] T. Geddert, I. Bialuch, W. Augustin, S. Scholl, Extending the induction period of crystallization fouling through surface coating, Heat Transfer Engineering 30 (2009) 868-875. 
[6] M. Vendel, A. C. Rasmuson, Mechanisms of initiation of incrustation, American Institute of Chemical Engineers Journal 43 (1997) 1300-1308.

[7] T. Geddert, W. Augustin, S. Scholl, Influence of Surface Defects and Aging of Coated Surfaces on Fouling Behavior, Heat Transfer Engineering 32 (2011) 300-306.

[8] M. Förster, W. Augustin, M. Bohnet, Influence of the adhesion force crystal/heat exchanger surface on fouling mitigation, Chemical Engineering and Processing: Process Intensification 38 (1999) 449-461.

[9] M. Raphael, S. Rohani, On-line estimation of solids concentrations and mean particle size using a turbidimetry method, Powder Technology 89 (1996) 157-163.

[10] M. Moscosa-Santillán, O. Bals, H. Fauduet, C. Porte, A. Delacroix, Study of batch crystallization and determination of an alternative temperature-time profile by on-line turbidity analysis - application to glycine crystallization, Chemical Engineering Science 55 (2000) 37593770 .

[11] M. S. N. Oliveira, A. W. Fitch, X.-W. Ni, A study of bubble velocity and bubble residence time in a gassed oscillatory baffled column - Effect of oscillation frequency, Chemical Engineering Research and Design 81 (2003) 233-242.

[12] Z. K. Nagy, K. Hungerbuhler, L. L. Simon, Comparison of external bulk video imaging with focused beam reflectance measurement and ultra-violet visible spectroscopy for metastable zone iden- 
tification in food and pharmaceutical crystallization processes, 2009. doi:10.1016/j.ces.2009.04.016.

[13] L. L. Simon, Z. K. Nagy, K. Hungerbuhler, Endoscopy-Based in Situ Bulk Video Imaging of Batch Crystallization Processes, Organic Process Research Development 13 (2009) 1254-1261.

[14] C. J. Brown, X.-W. Ni, Online Evaluation of Paracetamol Antisolvent Crystallization Growth Rate with Video Imaging in an Oscillatory Baffled Crystallizer, Crystal Growth \& Design 11 (2011) 719-725.

[15] Z. K. Nagy, G. Fevotte, H. Kramer, L. L. Simon, Recent advances in the monitoring, modelling and control of crystallization systems, Chemical Engineering Research and Design 91 (2013) 1903-1922.

[16] L. L. Simon, T. Merz, S. Dubuis, A. Lieb, K. Hungerbuhler, In-situ monitoring of pharmaceutical and specialty chemicals crystallization processes using endoscopy-stroboscopy and multivariate image analysis, Chemical Engineering Research and Design 90 (2012) 1847-1855.

[17] L. L. Simon, K. Abbou Oucherif, Z. K. Nagy, K. Hungerbuhler, Bulk video imaging based multivariate image analysis, process control chart and acoustic signal assisted nucleation detection, Chemical Engineering Science 65 (2010) 4983-4995.

[18] L. L. Simon, K. A. Oucherif, Z. K. Nagy, K. Hungerbuhler, Histogram matching, hypothesis testing, and statistical control-chart-assisted nucleation detection using bulk video imaging for optimal switching be- 
tween nucleation and seed conditioning steps, Industrial and Engineering Chemistry Research 49 (2010) 9932-9944.

[19] X.-W. Ni, Continuous Oscillatory Baffled Reactor Technology, Innovations in Pharmaceutical Technology (2006) 8-12.

[20] P. Stonestreet, A. P. Harvey, A Mixing-Based Design Methodology for Continuous Oscillatory Flow Reactors, Chemical Engineering Research and Design 80 (2002) 31-44.

[21] E. Manzurola, A. Apelblat, Solubilities of l-glutamic acid, 3-nitrobenzoic acid, p-toluic acid, calcium-l-lactate, calcium gluconate, magnesium-dlaspartate, and magnesium-l-lactate in water, The Journal of Chemical Thermodynamics 34 (2002) 1127-1136.

[22] H.-D. Belitz, W. Grosch, P. Schieberle, Food Chemistry, 4th ed., Springer, 2009.

[23] R. P. Adams, D. J. C. MacKay, Bayesian Online Changepoint Detection, Cambridge, UK, 2007.

[24] B. Amidan, T. Ferryman, S. Cooley, Data outlier detection using the Chebyshev theorem, 2005 IEEE Aerospace Conference (2005). 